\title{
The effects of timing of prophylaxis, type of anesthesia, and use of mechanical methods on outcome in major orthopedic surgery - subgroup analyses from 17,70I patients in the XAMOS study
}

\author{
This article was published in the following Dove Press journal: \\ Vascular Health and Risk Management \\ 18 May 2016 \\ Number of times this article has been viewed
}

Sylvia Haas'

Gerlind Holberg ${ }^{2}$

Reinhold Kreutz ${ }^{3}$

Michael Rud Lassen ${ }^{4}$

Lorenzo Mantovani ${ }^{5}$

Verena Haupt ${ }^{6}$

Kai Vogtländer ${ }^{7}$

Alexander GG Turpie ${ }^{8}$

'Formerly Technical University of Munich, ${ }^{2}$ Bayer HealthCare AG,

${ }^{3}$ Institut für Klinische Pharmakologie

und Toxikologie, Charité-

Universitätsmedizin, Berlin, Germany;

${ }^{4}$ Glostrup Hospital, University of

Copenhagen, Glostrup, Denmark;

${ }^{5}$ CESP-Center for Public Health

Research, University of Milan-Bicocca,

Monza, Italy; ${ }^{6}$ Bayer Vital GmbH,

Leverkusen, ${ }^{7}$ Bayer Pharma AG,

Wuppertal, Germany; ${ }^{8}$ Department of

Medicine, Hamilton Health Services,

Hamilton, ON, Canada
Correspondence: Sylvia Haas

Formerly Technical University of Munich,

Normannenstrasse 34a, 8I 925 Munich,

Germany

Tel +4989 917191

Fax +4989917177

Emailsylvia.haas@thromboscientific.com
Purpose: Real-world data on the use of rivaroxaban in the perioperative period in patients undergoing major orthopedic surgery are limited. Subsets of data from the Phase IV, noninterventional XAMOS study were analyzed to explore the potential influence of timing of the first thromboprophylactic dose, type of anesthesia, and concomitant mechanical prophylaxis on clinical outcomes in patients undergoing major orthopedic surgery in routine clinical practice.

Patients and methods: In XAMOS, 8,778 patients received rivaroxaban (10 mg once daily) and 8,635 received standard-of-care (SOC) pharmacological prophylaxis (safety population). Crude incidences of symptomatic thromboembolic and treatment-emergent bleeding events were analyzed according to timing of the first postoperative thromboprophylactic dose, use of general or neuraxial anesthesia, and use of mechanical prophylaxis with pharmacological thromboprophylaxis.

Results: In the rivaroxaban group, the incidences of symptomatic thromboembolic events were $0.7 \%, 1.0 \%$, and $0.7 \%$ in patients receiving the first thromboprophylactic dose at $\leq 6$ hours, $>6$ hours to $\leq 10$ hours, and $>10$ hours to $\leq 24$ hours after surgery, respectively. In the SOC group, the incidence of symptomatic thromboembolic events was slightly higher when the postoperative dose was given at $>10$ hours to $\leq 24$ hours $(1.8 \%$ vs $1.1 \%$ at $\leq 6$ hours and $1.3 \%$ at $>6$ hours to $\leq 10$ hours). The antithrombotic effect of rivaroxaban was maintained in comparison to the SOC group. The incidence of major bleeding (RECORD trial definition) was low and similar between the two treatment groups and was not influenced by timing of the first thromboprophylactic dose. Neuraxial anesthesia was used more than any other form of anesthesia for both hip and knee surgery; the effectiveness of rivaroxaban was not influenced by the type of anesthesia used. No spinal hematomas were reported in patients receiving neuraxial anesthesia in either treatment group. Use of mechanical thromboprophylaxis in addition to rivaroxaban or SOC pharmacological prophylaxis did not reduce the risk of thromboembolic events further.

Conclusion: The effectiveness and safety of rivaroxaban in patients undergoing major orthopedic surgery in routine clinical practice were maintained irrespective of timing of the first postoperative dose within 24 hours after surgery, the type of anesthesia, and the additional use of mechanical thromboprophylaxis.

Keywords: bleeding event, rivaroxaban, thromboprophylaxis, VTE prevention

\section{Introduction}

Patients undergoing major orthopedic surgery, such as elective hip and knee replacement surgery, are at high risk of venous thromboembolism (VTE), which can be 
reduced by pharmacological prophylaxis. ${ }^{1}$ Rivaroxaban, one of the non-vitamin $\mathrm{K}$ antagonist direct oral anticoagulants (DOACs), was shown to be more effective than enoxaparin regimens for the prevention of VTE after elective hip and knee replacement surgery, with a similar safety profile, in the Phase III RECORD trials. ${ }^{2-6}$ The effectiveness and safety of rivaroxaban in unselected patients in routine clinical practice were confirmed in XAMOS, a noninterventional, Phase IV study, which demonstrated that rivaroxaban was associated with a lower incidence of symptomatic thromboembolic events compared with standard-of-care (SOC) pharmacological thromboprophylaxis in patients undergoing major orthopedic surgery of the lower limbs. ${ }^{7}$

It has been suggested that the timing of the first postoperative dose of pharmacological thromboprophylaxis and the type of anesthesia used (ie, general vs neuraxial) during surgery may affect the clinical outcomes in patients undergoing major orthopedic surgery. ${ }^{8,9}$ In an earlier systematic review of 141 studies, neuraxial anesthesia was shown to significantly reduce mortality and the occurrence of deep vein thrombosis and pulmonary embolism. ${ }^{10}$ Guidelines also recommend the use of mechanical methods to prevent VTE, particularly in patients at a high risk of bleeding. ${ }^{1}$ However, the potential benefit of using mechanical thromboprophylaxis (such as compression stockings) in combination with pharmacological thromboprophylaxis is yet to be confirmed, because clear clinical evidence is lacking. ${ }^{1}$

As with the other DOACs approved for clinical use, real-life data on the use of rivaroxaban in the perioperative period in patients undergoing major orthopedic surgery are limited - such as data regarding the timing of the first dose of pharmacological thromboprophylaxis, the use of neuraxial anesthesia, and the concomitant use of mechanical prophylaxis in daily practice. The aim of the subanalyses of the XAMOS study was to describe these parameters and explore their potential influence on clinical outcomes (focusing on symptomatic thromboembolic and bleeding events) in patients undergoing major orthopedic surgery of the lower limbs in routine clinical practice.

\section{Patients and methods}

\section{Study population and clinical outcomes}

XAMOS was a Phase IV, international, noninterventional, open-label cohort study. Eligible patients were aged $\geq 18$ years, with planned hip or knee replacement surgery (or fracture-related orthopedic surgery in countries where rivaroxaban is indicated) and in whom a decision on pharmacological thromboprophylaxis had already been made.
Exclusion criteria were based on the contraindications in the approved local product information (Summary of Product Characteristics), and written informed consent was obtained from patients in countries where necessary. ${ }^{7}$ This study is registered with ClinicalTrials.gov (NCT00831714). The study protocol was approved by the European Medicines Agency (EMA) and the appropriate independent Ethics Committee or an Independent Review Board where required. The study was conducted in accordance with Good Epidemiological Practice guidance and was supervised by a Steering Committee.

The type, dose, and duration of pharmacological thromboprophylaxis were decided by the treating physician prior to patient enrollment. Out of the 17,413 patients included in the safety population, 8,778 patients received rivaroxaban (10 mg once daily) and 8,635 received SOC (including, but not limited to, low-molecular-weight heparins [81.7\%], fondaparinux, and dabigatran etexilate). ${ }^{7}$ The types of anesthesia used, the timing of the first dose of thromboprophylaxis, and the use of mechanical prophylaxis (such as elastic stockings and intermittent pneumatic compression [IPC]) were also recorded.

Data were collected on adverse events, including symptomatic thromboembolic events and bleeding events, and coded according to the standardized Medical Dictionary for Regulatory Activities (version 14.0). ${ }^{7}$ Symptomatic thromboembolic events occurring during the entire study period were identified and adjudicated in a treatment-blinded fashion. Data collected on bleeding events were differentiated as major and nonmajor bleeding events. The primary safety outcome was major bleeding as defined in the RECORD trials, ${ }^{5}$ that is, clinically overt bleeding that was fatal, occurred in a critical organ, necessitated reoperation, or was outside of the surgical site and was associated with a fall in hemoglobin of $\geq 2 \mathrm{~g} / \mathrm{dL}$ or required a transfusion of $\geq 2$ units of blood. In addition, major bleeding events were defined according to the European Medicines Agency (EMA) guidelines, which are similar to the RECORD major bleeding definition but with the inclusion of bleeding warranting treatment cessation and surgical-site bleeding events associated with a fall in hemoglobin of $\geq 2 \mathrm{~g} / \mathrm{dL}$ or leading to a transfusion of $\geq 2$ units of blood or packed cells. ${ }^{11}$ Treatment-emergent events were defined as those occurring on or after the day of the first dose and within 48 hours after the last dose of thromboprophylactic drug.

\section{Data analyses}

Cumulative crude incidences were calculated for the rivaroxaban and SOC groups in the safety population, which included 
patients who received at least one dose of rivaroxaban or SOC. Thromboembolic events were those that occurred within 3 months of surgery, whereas bleeding events presented were treatment-emergent events.

In this analysis, data were analyzed according to the timing of the first postoperative dose of rivaroxaban and SOC thromboprophylaxis (ie, $\leq 6$ hours, $>6$ hours to $\leq 10$ hours, and $>10$ hours to $\leq 24$ hours after surgery), type of anesthesia used (ie, general, neuraxial, peripheral [including peripheral blocks or local infiltration], combinations, and unknown/others), and whether mechanical prophylaxis (eg, compression stockings and IPC) was used concomitantly with pharmacological thromboprophylaxis.

The analyses are of an exploratory, descriptive nature, with the purpose to identify directional trends based on odds ratios (ORs) given with 95\% confidence intervals (CIs), in context of the overall results.

\section{Results \\ Outcomes by timing of the first postoperative dose of thromboprophylaxis}

Information on the timing of the first postoperative dose was available for most patients in XAMOS. Almost all patients (94\%) started antithrombotic therapy (rivaroxaban or SOC) within 24 hours after surgery. Of the 8,778 patients who received rivaroxaban, 4,985 (56.8\%) received their first dose $>6$ hours to $\leq 10$ hours after surgery. There were $1,541(17.6 \%)$ and $1,758(20.0 \%)$ patients who initiated rivaroxaban $\leq 6$ hours and $>10$ hours to $\leq 24$ hours after surgery, respectively. In the SOC group, the first postoperative dose was given at $\leq 6$ hours, $>6$ hours to $\leq 10$ hours, and $>10$ hours to $\leq 24$ hours in 2,189 (25.4\%), 3,553 (41.1\%), and 2,225 (25.8\%) patients, respectively. In both treatment groups, some patients received their first postoperative dose at $>24$ hours.

The antithrombotic effect of rivaroxaban was well maintained in patients who received their first rivaroxaban dose up to 24 hours after surgery. The incidences of symptomatic thromboembolic events and treatment-emergent major bleeding events in patients who received the first dose of rivaroxaban at $\leq 6$ hours, $>6$ hours to $\leq 10$ hours, and $>10$ hours to $\leq 24$ hours after surgery were similar to those in the overall rivaroxaban patient population (Figure 1A). In the SOC group, the incidences of symptomatic thromboembolic events were similar between those receiving the first dose of SOC at $\leq 6$ hours and at $>6$ hours to $\leq 10$ hours after surgery but were slightly higher in those who had their first dose at $>10$ hours to $\leq 24$ hours. The incidences of treatment-emergent major bleeding events were similar when the first dose of SOC was initiated at $\leq 6$ hours or $>6$ hours to $\leq 10$ hours but appeared to be lower for those receiving the first dose at $>10$ hours to $\leq 24$ hours after surgery (Figure 1B).

For patients who received their first postoperative dose $\leq 6$ hours after surgery, symptomatic thromboembolic events occurred in $0.7 \%$ and $1.1 \%$ for the rivaroxaban and SOC groups, respectively (OR $=0.62 ; 95 \%$ CI $0.31-1.27$ ) (Figure 2A). In those who initiated rivaroxaban $>6$ hours to $\leq 10$ hours after surgery, the incidence was $1.0 \%$ for rivaroxaban and $1.3 \%$ for SOC (OR $=0.74 ; 95 \%$ CI $0.49-1.11$ ). The effectiveness of rivaroxaban was maintained in patients who received their first rivaroxaban dose $>10$ hours to $\leq 24$ hours after surgery $(0.7 \%$ vs $1.8 \%$ for rivaroxaban and SOC; OR $=0.42 ; 95 \%$ CI $0.22-0.78$ ) (Figure 2A). The incidence of treatment-emergent major bleeding (RECORD definition) was low and similar between the two treatment groups and was not influenced by the timing of the first dose of thromboprophylaxis; major bleeding occurred in $0.3 \%$, $0.5 \%$, and $0.3 \%$ of patients in the rivaroxaban group and in $0.4 \%, 0.4 \%$, and $0.2 \%$ of patients in the SOC group when the first dose was given at $\leq 6$ hours, $>6$ hours to $\leq 10$ hours, and $>10$ hours to $\leq 24$ hours after surgery, respectively (Figure 2B). When the EMA definition of major bleeding was used, the incidence was higher (compared with the RECORD definition) but similar between the two treatment groups irrespective of the timing of the first dose, except for those who initiated rivaroxaban or SOC $>10$ hours to $\leq 24$ hours after surgery $(1.8 \%$ for rivaroxaban and $0.9 \%$ for SOC; OR $=1.98 ; 95 \%$ CI 1.12-3.48; Figure 2B). A higher incidence of any treatment-emergent bleeding events was reported for the rivaroxaban group compared with the SOC group in patients who received their first thromboprophylaxis dose at $>6$ hours to $\leq 10$ hours $(4.4 \%$ vs $3.2 \%$; OR $=1.39 ; 95 \%$ CI $1.11-1.75)$ and $>10$ hours to $\leq 24$ hours $(5.7 \%$ vs $3.0 \%$; OR $=1.97 ; 95 \%$ CI 1.44-2.71) after surgery, but not at $\leq 6$ hours after surgery (3.8\% vs $3.3 \%$; OR $=1.15$; 95\% CI 0.81-1.64).

\section{Outcomes by types of anesthesia used}

In total, 6,373 patients (36.6\%) received general anesthesia, $10,355(59.5 \%)$ received neuraxial anesthesia (spinal or epidural), $113(0.6 \%)$ received peripheral anesthesia, and $523(3 \%)$ received combinations $(0.3 \%$ were unknown/ missing and received other combinations/missing) (Figure $3)$. Neuraxial anesthesia was used more than any other form of anesthesia for both hip and knee surgery. General anesthesia was used more often in hip surgery than knee surgery. The types of anesthesia used were similar between the two 

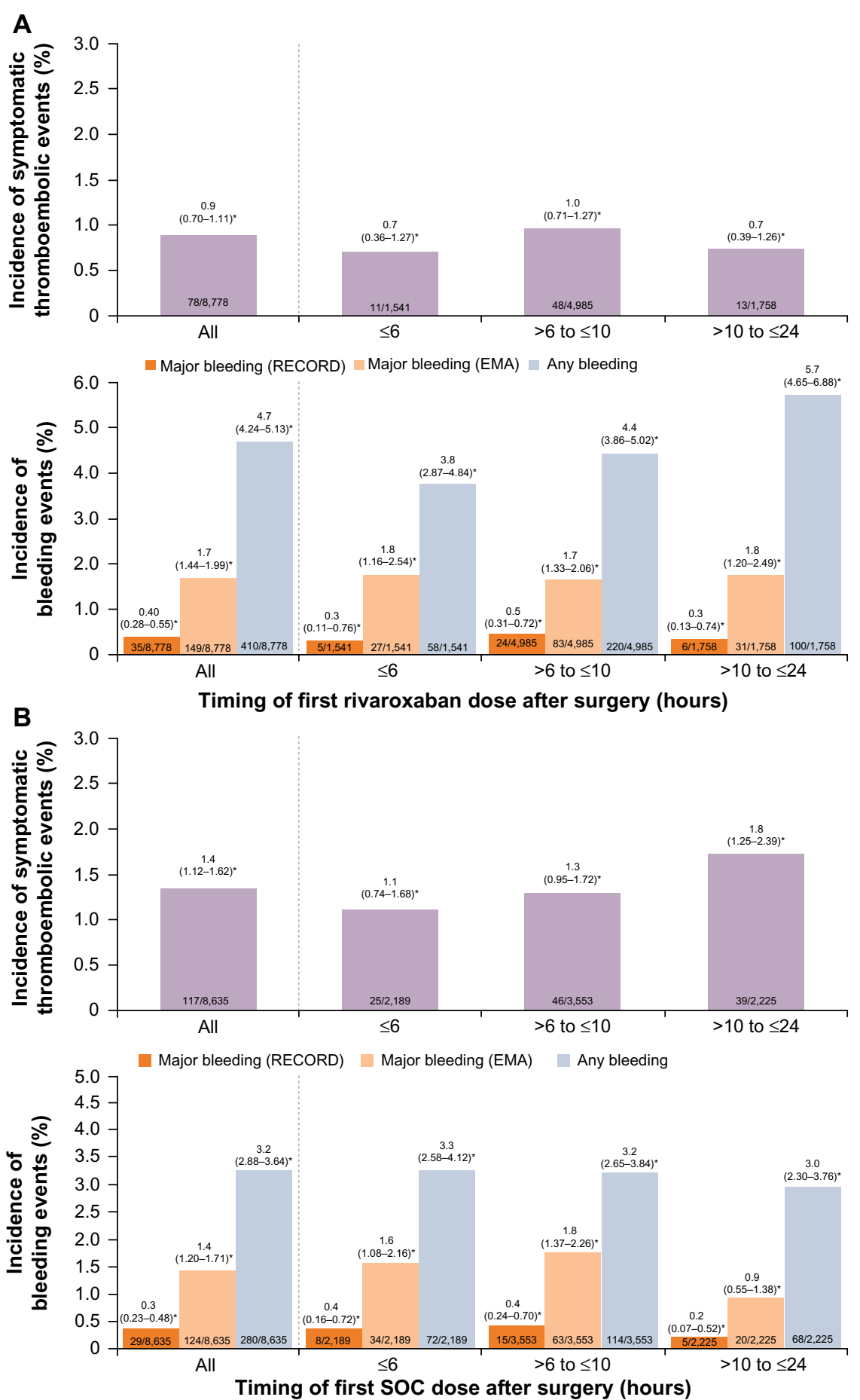

Figure I Incidences of symptomatic thromboembolic events and treatment-emergent major bleeding events by timing of first dose of prophylactic therapy.

Notes: (A) Rivaroxaban. (B) Standard of care. ${ }^{* 95 \%}$ confidence interval. Results are presented for the safety population (patients who received at least one dose of rivaroxaban or SOC).

Abbreviations: EMA, European Medicines Agency; SOC, standard of care.

treatment groups (Figure 3). Outcomes are reported here only in patients who received general or neuraxial anesthesia and are compared with the overall XAMOS cohort. The overall results of the XAMOS study showed a lower incidence of symptomatic thromboembolic events with rivaroxaban than with SOC, with similar incidences of treatment-emergent major bleeding events (safety population). ${ }^{7}$ In patients who received general anesthesia, symptomatic thromboembolic events occurred in $1.2 \%(39 / 3,240)$ and $1.7 \%(53 / 3,133)$ for the rivaroxaban and the $\mathrm{SOC}$ groups, respectively $(\mathrm{OR}=0.71$; $95 \%$ CI 0.47-1.07). In patients receiving neuraxial anesthesia, symptomatic thromboembolic events occurred in $0.6 \%$ 
A

\begin{tabular}{|c|c|c|c|c|c|}
\hline \multirow{2}{*}{$\begin{array}{l}\text { Timing of first dose } \\
\text { after surgery (hours) }\end{array}$} & \multirow{2}{*}{$\begin{array}{c}\text { Rivaroxaban } \\
\quad \%(\mathrm{n} / \mathrm{N})\end{array}$} & \multirow{2}{*}{$\begin{array}{l}\text { SOC } \\
\%(n / N)\end{array}$} & \multirow[t]{2}{*}{ OR $(95 \% \mathrm{Cl})$} & \multicolumn{2}{|c|}{ OR $(95 \% \mathrm{Cl})$} \\
\hline & & & & $\begin{array}{c}\text { Favors } \\
\text { rivaroxaban }\end{array}$ & $\begin{array}{c}\text { Favors } \\
\text { SOC }\end{array}$ \\
\hline$\leq 6$ & $0.7(11 / 1,541)$ & $1.1(25 / 2,189)$ & $0.62(0.31-1.27)$ & & \\
\hline$>6$ to $\leq 10$ & $1.0(48 / 4,985)$ & $1.3(46 / 3,553)$ & $0.74(0.49-1.11)$ & $\mapsto$ & \\
\hline$>10$ to $\leq 24$ & $0.7(13 / 1,758)$ & $1.8(39 / 2,225)$ & $0.42(0.22-0.78)$ & $\longmapsto$ & \\
\hline
\end{tabular}

B

\begin{tabular}{|c|c|c|c|c|c|c|}
\hline \multirow[t]{2}{*}{ Definition } & \multirow{2}{*}{$\begin{array}{l}\text { Timing of first dose } \\
\text { after surgery (hours) }\end{array}$} & \multirow{2}{*}{$\begin{array}{l}\text { Rivaroxaban } \\
\%(n / N)\end{array}$} & \multirow{2}{*}{$\begin{array}{c}\text { SOC } \\
\%(n / N)\end{array}$} & \multirow[t]{2}{*}{ OR $(95 \% \mathrm{Cl})$} & \multicolumn{2}{|c|}{ OR $(95 \% \mathrm{CI})$} \\
\hline & & & & & $\begin{array}{c}\text { Favors } \\
\text { rivaroxaban }\end{array}$ & $\begin{array}{l}\text { Favors } \\
\text { SOC }\end{array}$ \\
\hline \multirow{3}{*}{$\begin{array}{l}\text { Major } \\
\text { bleeding } \\
\text { (RECORD) }\end{array}$} & $\leq 6$ & $0.3(5 / 1,541)$ & $0.4(8 / 2,189)$ & $0.89(0.29-2.72)$ & $\mapsto$ & H \\
\hline & $>6$ to $\leq 10$ & $0.5(24 / 4,985)$ & $0.4(15 / 3,553)$ & $1.14(0.60-2.18)$ & & \\
\hline & $>10$ to $\leq 24$ & $0.3(6 / 1,758)$ & $0.2(5 / 2,225)$ & $1.52(0.46-4.99)$ & $\vdash$ & -1 \\
\hline \multirow{3}{*}{$\begin{array}{l}\text { Major } \\
\text { bleeding } \\
\text { (EMA) }\end{array}$} & $\leq 6$ & $1.8(27 / 1,541)$ & $1.6(34 / 2,189)$ & $1.1(0.68-1.88)$ & & \\
\hline & $>6$ to $\leq 10$ & $1.7(83 / 4,985)$ & $1.8(63 / 3,553)$ & $0.9(0.67-1.31)$ & & \\
\hline & $>10$ to $\leq 24$ & $1.8(31 / 1,758)$ & $0.9(20 / 2,225)$ & $2.0(1.12-3.48)$ & & -1 \\
\hline \multirow{3}{*}{$\begin{array}{l}\text { Any } \\
\text { bleeding }\end{array}$} & $\leq 6$ & $3.8(58 / 1,541)$ & $3.3(72 / 2,189)$ & $1.15(0.81-1.64)$ & & \\
\hline & $>6$ to $\leq 10$ & $4.4(220 / 4,985)$ & $3.2(114 / 3,553)$ & $1.39(1.11-1.75)$ & & \\
\hline & $>10$ to $\leq 24$ & $5.7(100 / 1,758)$ & $3.0(66 / 2,225)$ & $1.97(1.44-2.71)$ & & \\
\hline
\end{tabular}

Figure 2 Incidences of events by timing of first dose of thromboprophylaxis.

Notes: (A) Symptomatic thromboembolic events. (B) Treatment-emergent bleeding events. Results are presented for the safety population (patients who received at least one dose of rivaroxaban or SOC).

Abbreviations: $\mathrm{Cl}$, confidence interval; EMA, European Medicines Agency; OR, odds ratio; SOC, standard of care.

$(31 / 5,187)$ of patients in the rivaroxaban group and in $1.1 \%(55 / 5,168)$ of patients in the SOC group (OR $=0.56$; 95\% CI 0.36-0.87) (Figure 4A). Overall, the incidence of symptomatic thromboembolic events appeared to be numerically higher after knee surgery (1.4\%) than after hip surgery $(0.9 \%)$ but, in general, the type of anesthesia used did not seem to influence the effect of rivaroxaban (Table 1).

Similar incidences of treatment-emergent major bleeding events were observed between the two treatment groups and were not influenced by the type of anesthesia used (Figure 4B). In patients who received general anesthesia, the incidence of treatment-emergent major bleeding (RECORD definition) was $0.4 \%(13 / 3,240)$ in the rivaroxaban group and $0.4 \%(14 / 3,133)$ in the SOC group $(\mathrm{OR}=0.90 ; 95 \% \mathrm{CI}$
0.42-1.91). In patients who received neuraxial anesthesia, treatment-emergent major bleeding (RECORD definition) occurred in $0.4 \%(21 / 5,187)$ of patients in the rivaroxaban group and $0.3 \%(15 / 5,168)$ of patients in the SOC group $(\mathrm{OR}=1.40 ; 95 \% \mathrm{CI} 0.72-2.71)$. When the EMA definition of major bleeding was used, the incidences were similar between the treatment groups and between patients who received general and neuraxial anesthesia: $1.8 \%$ for the rivaroxaban group versus $1.7 \%$ for the SOC group (OR $=1.06$; $95 \%$ CI $0.73-1.54)$ in patients receiving general anesthesia and $1.6 \%$ versus $1.2 \%(\mathrm{OR}=1.36 ; 95 \% \mathrm{CI} 0.98-1.90)$ in those receiving neuraxial anesthesia (Figure 4B). There were no spinal hematomas reported in patients receiving neuraxial anesthesia (including the use of indwelling catheter) in either treatment group in this study. 


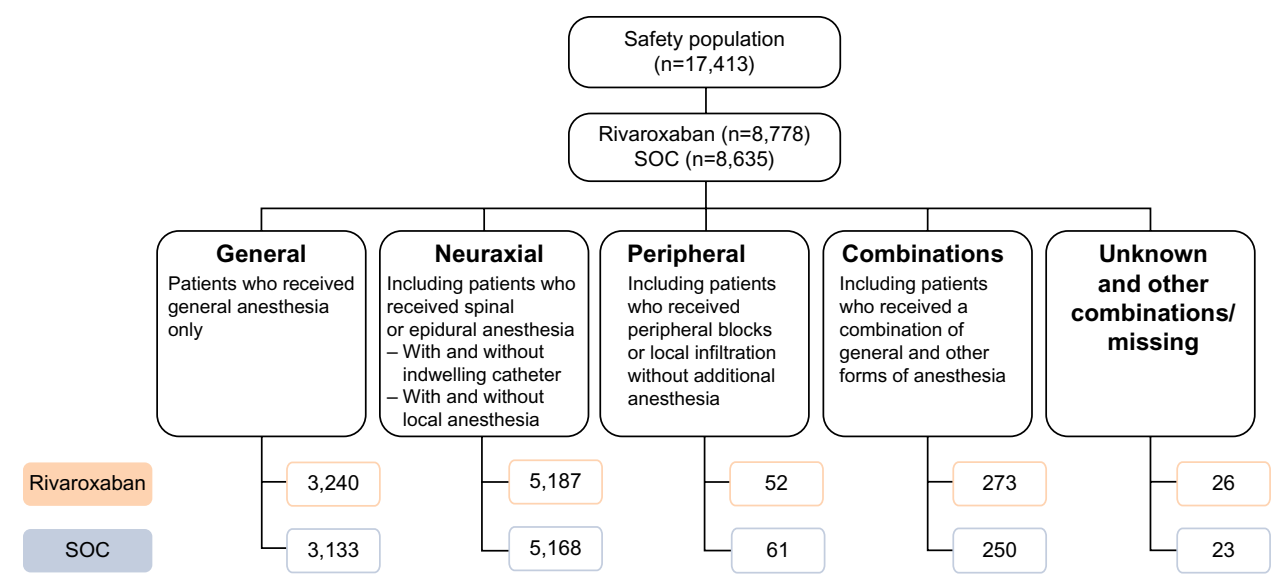

Figure 3 Types of anesthesia used in the XAMOS study. Abbreviation: $\mathrm{SOC}$, standard of care.

\section{Outcomes by use of mechanical thromboprophylaxis}

The percentage of patients using mechanical methods alongside pharmacological thromboprophylaxis was similar between the rivaroxaban and the SOC groups $(62 \%$ and $59 \%$, respectively). These methods were primarily compression stockings (92\%) and IPC (12\%). Postoperative use of mechanical thromboprophylaxis varied between regions, with use ranging from 18\% in Scandinavia to $70 \%$ in Asia.

The use of mechanical thromboprophylaxis did not appear to further reduce the risk of thromboembolic events. Symptomatic thromboembolic events occurred in $0.8 \%$ $(45 / 5,430)$ and $1.4 \%(71 / 5,107)$ in the rivaroxaban and SOC groups, respectively, for patients who also used mechanical thromboprophylaxis compared with $1.0 \%(33 / 3,348)$ and $1.3 \%(46 / 3,528)$ for rivaroxaban and SOC groups, respectively, for nonusers (Figure 5). The overall incidences of treatment-emergent adverse events, as well as those for nonbleeding-related adverse events, including skin disorders, were similar between the two treatment groups, irrespective of the use of mechanical prophylaxis (data not shown).

\section{Discussion}

Published data on the use of DOACs in everyday clinical practice are limited, particularly with regard to the potential influence of timing of the first dose of thromboprophylaxis in the perioperative period, as well as their use under different types of anesthesia and with or without mechanical prophylaxis. Data on these parameters were recorded in the noninterventional XAMOS study, and the outcomes of these subanalyses of XAMOS data are consistent with the overall findings of the study, which confirmed the effectiveness and safety of rivaroxaban in unselected patients in routine clinical practice. ${ }^{?}$

Previous studies have suggested that the timing of the first dose of thromboprophylaxis may have an impact on clinical outcomes, such as bleeding and thromboembolic events. ${ }^{9}{ }^{12}$ In a meta-analysis of four randomized trials in patients undergoing major orthopedic surgery, the timing of the first dose of fondaparinux was associated with the incidence of major bleeding events, and patients who initiated fondaparinux within 6 hours after surgery had a higher incidence of overt bleeding. ${ }^{9}$ The label for rivaroxaban recommends starting rivaroxaban 6-10 hours after surgery when hemostasis has been established. ${ }^{13}$ These subanalyses of the data from XAMOS showed that, in routine clinical practice, only $56.8 \%$ of patients received their first dose of rivaroxaban within the recommended time frame of 6-10 hours after surgery. ${ }^{13}$ Moreover, patients who initiated rivaroxaban $\leq 6$ hours after surgery did not have a higher incidence of major bleeding

Table I Incidence of symptomatic thromboembolic events by type of surgery and anesthesia

\begin{tabular}{|c|c|c|c|c|c|c|}
\hline \multirow[t]{2}{*}{ Type of anesthesia } & \multicolumn{3}{|l|}{ Hip surgery } & \multicolumn{3}{|l|}{ Knee surgery } \\
\hline & Rivaroxaban & soc & Total & Rivaroxaban & soc & Total \\
\hline All, \% (n/N) & $0.6(28 / 4,688)$ & I.2(55/4,637) & $0.9(83 / 9,325)$ & I.2 (50/4,052) & $1.6(62 / 3,950)$ & $1.4(\mid \mathrm{I} / 2 / 8,002)$ \\
\hline General, \% (n/N) & $0.9(19 / 2,026)$ & I.2 (24/I,940) & I.I $(43 / 3,966)$ & $1.7(20 / 1,194)$ & $2.5(29 / 1,164)$ & $2.1(49 / 2,358)$ \\
\hline Neuraxial, \% (n/N) & $0.3(8 / 2,550)$ & I.0 (27/2,596) & $0.7(35 / 5,146)$ & $0.9(23 / 2,622)$ & I.I $(28 / 2,557)$ & $1.0(5 \mathrm{I} / 5, \mid 79)$ \\
\hline
\end{tabular}

Note: Results are presented for the safety population (patients who received at least one dose of rivaroxaban or SOC).

Abbreviation: SOC, standard of care. 
A

\begin{tabular}{|c|c|c|c|c|c|c|}
\hline & & Rivaroxaban & SOC & OR $(95 \% \mathrm{Cl})$ & OR ( & \\
\hline & & $\%(n / N)$ & $\%(n / N)$ & & $\begin{array}{c}\text { Favors } \\
\text { rivaroxaban }\end{array}$ & $\begin{array}{c}\text { Favors } \\
\text { SOC }\end{array}$ \\
\hline Any symptomatic & All patients & $0.9(78 / 8,778)$ & $1.4(117 / 8,635)$ & $0.65(0.49-0.87)$ & & \\
\hline & General & $1.2(39 / 3,240)$ & $1.7(53 / 3,133)$ & $0.71(0.47-1.07)$ & & \\
\hline & Neuraxial & $0.6(31 / 5,187)$ & $1.1(55 / 5,168)$ & $0.56(0.36-0.87)$ & & \\
\hline Symptomatic & All patients & $0.7(57 / 8,778)$ & $1.0(88 / 8,635)$ & $0.63(0.45-0.89)$ & & \\
\hline events & General & $0.8(26 / 3,240)$ & $1.4(43 / 3,133)$ & $0.58(0.36-0.95)$ & & \\
\hline & Neuraxial & $0.4(23 / 5,187)$ & $0.8(40 / 5,168)$ & $0.57(0.34-0.96)$ & & \\
\hline Symptomatic & All patients & $0.2(20 / 8,778)$ & $0.3(29 / 8,635)$ & $0.68(0.38-1.20)$ & & \\
\hline events & General & $0.4(12 / 3,240)$ & $0.3(10 / 3,133)$ & $1.16(0.50-2.69)$ & & † \\
\hline & Neuraxial & $0.2(8 / 5,187)$ & $0.3(15 / 5,168)$ & $0.53(0.22-1.25)$ & & \\
\hline
\end{tabular}

B

\begin{tabular}{|c|c|c|c|c|c|c|}
\hline & & Rivaroxaban & SOC & OR $(95 \% \mathrm{Cl})$ & OR (9 & \\
\hline & & & & & $\begin{array}{c}\text { Favors } \\
\text { rivaroxaban }\end{array}$ & $\begin{array}{c}\text { Favors } \\
\text { SOC }\end{array}$ \\
\hline \multirow{3}{*}{$\begin{array}{l}\text { Major bleeding } \\
\left.\text { (RECORD }^{\text {(Refinition) }}\right)^{\mathrm{a}}\end{array}$} & All patients & $0.4(35 / 8,778)$ & $0.3(29 / 8,635)$ & $1.19(0.73-1.95)$ & & \\
\hline & General & $0.4(13 / 3,240)$ & $0.4(14 / 3,133)$ & $0.90(0.42-1.91)$ & & \\
\hline & Neuraxial & $0.4(21 / 5,187)$ & $0.3(15 / 5,168)$ & $1.40(0.72-2.71)$ & & - \\
\hline \multirow{3}{*}{$\begin{array}{l}\text { Major bleeding } \\
\text { (EMA definition) }^{\text {a }}\end{array}$} & All patients & $1.7(149 / 8,778)$ & $1.4(124 / 8,635)$ & $1.19(0.93-1.51)$ & & \\
\hline & General & $1.8(58 / 3,240)$ & $1.7(53 / 3,133)$ & $1.06(0.73-1.54)$ & & \\
\hline & Neuraxial & $1.6(83 / 5,187)$ & $1.2(61 / 5,168)$ & $1.36(0.98-1.90)$ & & \\
\hline \multirow[t]{3}{*}{ Any bleeding ${ }^{a}$} & All patients & $4.7(410 / 8,778)$ & $3.2(280 / 8,635)$ & $1.46(1.25-1.71)$ & & \\
\hline & General & $4.2(136 / 3,240)$ & $3.1(98 / 3,133)$ & $1.36(1.04-1.77)$ & & \\
\hline & Neuraxial & $4.9(254 / 5,187)$ & $3.2(163 / 5,168)$ & $1.58(1.29-1.93)$ & & \\
\hline
\end{tabular}

Figure 4 Incidences of events by type of anesthesia.

Notes: (A) Symptomatic thromboembolic events. (B) Bleeding events. Results are presented for the safety population (patients who received at least one dose of rivaroxaban or SOC). ${ }^{a}$ Treatment emergent.

Abbreviations: $\mathrm{Cl}$, confidence interval; EMA, European Medicines Agency; OR, odds ratio; SOC, standard of care.

events (RECORD definition: 0.3\%) compared with those who had the first dose $>6$ hours to $\leq 10$ hours after surgery $(0.5 \%)$. Similarly, in the SOC group, the incidences of major bleeding were similar between patients who received their first dose $\leq 6$ hours after surgery $(0.4 \%)$ and $>6$ hours to $\leq 10$ hours after surgery $(0.4 \%)$. However, owing to the small patient numbers in this category and the nature of the noninterventional study, a definitive conclusion that earlier initiation of pharmacological thromboprophylaxis (ie, within 6 hours after surgery before hemostasis is established) has no 


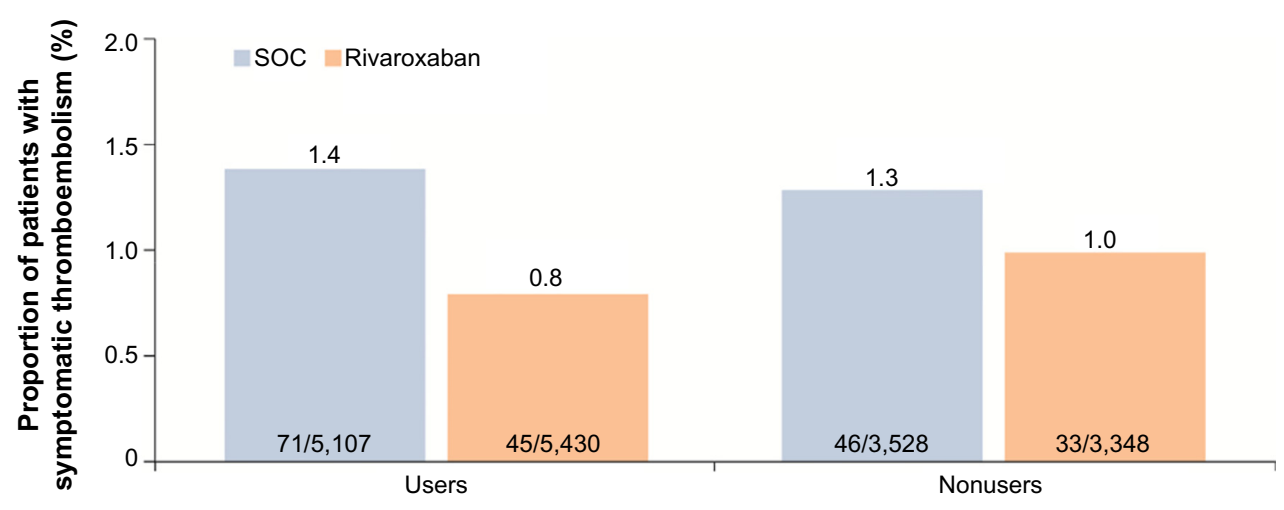

Figure 5 Incidences of symptomatic thromboembolic events in patients receiving rivaroxaban or SOC with and without the use of mechanical thromboprophylaxis. Note: Results are presented for the safety population (patients who received at least one dose of rivaroxaban or SOC). Abbreviation: SOC, standard of care.

impact on the risk of bleeding cannot be made based on these data. Patients receiving rivaroxaban initiated at $>10$ hours to $\leq 24$ hours after surgery (but not those starting at $\leq 6$ hours or $>6$ hours to $\leq 10$ hours after surgery) had a higher incidence of major bleeding when using the EMA definition $(1.8 \%$ vs $0.9 \%)$. On the basis of the overall study results of XAMOS, it is likely that this was driven by the incidence of bleeding leading to treatment cessation; although EMA major bleeding occurred more frequently in the rivaroxaban group in XAMOS, incidences were the same for both treatment groups when bleeding leading to treatment cessation was excluded. ${ }^{7}$ Most patients started rivaroxaban or SOC within 24 hours after surgery and, importantly, the effectiveness of rivaroxaban was not compromised when rivaroxaban was initiated $>10$ hours to $\leq 24$ hours after surgery: the incidence of symptomatic thromboembolic events was $0.7 \%$ in the rivaroxaban group compared with $1.8 \%$ in the SOC group ( $\mathrm{OR}=0.42 ; 95 \%$ CI $0.22-0.78)$. These data suggest that in certain patients or clinical circumstances, such as in patients who are unable to take oral drugs (eg, due to postoperative vomiting) or to comply with local hospital routines, a slight delay in initiating rivaroxaban (ie, beyond the recommended 6-10 hours after surgery but within 24 hours) may not compromise the effectiveness of rivaroxaban in preventing thromboembolic events in these patients.

There have been suggestions that the type of anesthesia used may potentially influence clinical outcomes. ${ }^{8}$ For example, patients receiving anticoagulants and undergoing neuraxial anesthesia may have an increased risk of spinal hematoma. ${ }^{14}$ In the Phase III RECORD trials, epidural catheter use was permitted, which is in contrast with other studies involving DOACs..$^{15}$ In this subanalysis of the XAMOS study, rivaroxaban showed a favorable benefit-risk profile compared with SOC that was consistent irrespective of the type of anesthesia used. This extended to patients receiving epidural anesthesia with indwelling catheters. Consistent with the findings of the RECORD trials, ${ }^{15}$ no cases of compressive spinal hematoma were reported in a population of $>17,000$ patients in daily practice. Overall, the incidence of symptomatic thromboembolic events appeared to be higher after knee surgery $(1.4 \%)$ than after hip surgery $(0.9 \%)$ but, in general, the type of anesthesia used did not seem to influence the effect of rivaroxaban. A numerically lower incidence of symptomatic thromboembolic events was observed in both treatment groups in patients who received neuraxial anesthesia compared with patients who received general anesthesia $(0.6 \%$ vs $1.2 \%$ in the rivaroxaban group and $1.1 \%$ vs $1.7 \%$ in the SOC group). Although earlier studies have indicated that neuraxial anesthesia was associated with a lower incidence of VTE, ${ }^{8}$ a systematic review of randomized controlled trials since 1990 concluded that there was insufficient evidence to determine whether anesthetic technique influenced mortality, cardiovascular morbidity, or the incidence of VTE when using thromboprophylaxis. ${ }^{16}$ Thus, our finding of lower incidence of symptomatic thromboembolic events in patients operated under neuraxial anesthesia requires further confirmation.

Mechanical thromboprophylaxis (eg, compression stockings and IPC) increases venous flow and reduces stasis within the veins and is recommended in guidelines for the prevention of VTE, primarily for patients at high risk of bleeding. ${ }^{1}$ In routine clinical practice, mechanical methods, such as stockings, are commonly prescribed in addition to pharmacological thromboprophylaxis. Unlike pharmacological thromboprophylaxis, clinical evidence on the benefit of using mechanical prophylaxis for the prevention of VTE is less clear and is questionable. In the XAMOS study, more than half of all patients used mechanical thromboprophylaxis in routine clinical practice, but use varied markedly between 
regions. Data from this subanalysis showed that the use of mechanical methods (in conjunction with rivaroxaban or SOC thromboprophylaxis) had no apparent influence on the incidence of symptomatic thromboembolic events compared with using rivaroxaban or SOC thromboprophylaxis alone. No additional reduction in the incidence of symptomatic thromboembolic events was observed with the use of mechanical methods. However, there might be specific reasons for the chosen mechanical method, but no further information is available in XAMOS. Nevertheless, the effectiveness of rivaroxaban compared with SOC was retained irrespective of the use of mechanical thromboprophylaxis. Data from this subanalysis are consistent with a recent meta-analysis of data from four studies in 1,171 patients undergoing trauma and elective orthopedic surgery, in which no significant benefit of anti-embolic stockings was shown in reducing the risk of VTE when used together with pharmacological thromboprophylaxis compared with pharmacological thromboprophylaxis alone. ${ }^{17}$ Therefore, data from this subanalysis raise further questions regarding the added value of mechanical prophylaxis used in combination with pharmacological prophylaxis. However, other meta-analyses have shown additional benefits of IPC in combination with pharmacological thromboprophylaxis compared with IPC or pharmacological thromboprophylaxis alone. ${ }^{18,19}$

It should be noted that these subanalyses are of an exploratory, descriptive nature, and the parameters described here reflect physicians' preference and/or local protocols in daily practice (eg, the use of neuraxial instead of general anesthesia). In addition, XAMOS was a noninterventional, open-label study; unlike randomized controlled trials, which use strict inclusion and exclusion criteria and may involve more comprehensive levels of medical care, noninterventional studies may result in selection bias and over- or underreporting of adverse events. For example, it has been suggested that there may be an increase in adverse event reporting after the introduction of the new drug, owing to higher levels of physician vigilance when comparing a new drug with established therapies (known as the Weber effect). ${ }^{20}$ This could have influenced the reported frequency of adverse events in XAMOS, including minor bleeding events. Nevertheless, data from these subanalyses of the XAMOS study provide valuable information on thromboprophylaxis in unselected patients in daily practice.

\section{Conclusion}

The results from these subanalyses confirm the effectiveness and safety of rivaroxaban in patients undergoing major orthopedic surgery in routine clinical practice, which were maintained irrespective of the timing of the first postoperative dose within 24 hours after surgery, the type of anesthesia used, and the use of mechanical thromboprophylaxis.

\section{Acknowledgments}

The information contained in this paper is presented on behalf of the XAMOS Investigators. The authors would like to acknowledge Yong-Ling Liu, who provided editorial assistance with funding from Bayer Pharma AG.

\section{Disclosure}

S Haas was a consultant for Aspen, Bayer HealthCare Pharmaceuticals, Boehringer Ingelheim, Bristol-Myers Squibb, Daiichi Sankyo, Pfizer, and Sanofi. R Kreutz has been a consultant for Bayer HealthCare Pharmaceuticals, BerlinChemie, Bristol-Myers Squibb, Daiichi Sankyo Pharma, Menarini, Merck, Trommsdorff, and Servier. MR Lassen has been a consultant for Bristol-Myers Squibb, DepuySynthes, Medtronic, Pfizer, and Sanofi-Aventis and has been paid for educational presentations from Bayer HealthCare Pharmaceuticals and Janssen Pharmaceutical Research \& Development, LLC. L Mantovani has been a consultant for Bayer HealthCare Pharmaceuticals and has received grants from Boehringer Ingelheim, Bristol-Myers Squibb, Daiichi Sankyo, and Pfizer. G Holberg, V Haupt, and K Vogtländer are employees of Bayer HealthCare Pharmaceuticals. AGG Turpie has been a consultant for Astellas, Bayer HealthCare, Janssen Pharmaceutical Research \& Development, LLC, Portola, and Takeda. The authors report no other conflicts of interest in this work.

\section{References}

1. Falck-Ytter Y, Francis CW, Johanson NA, et al. Prevention of VTE in orthopedic surgery patients: antithrombotic therapy and prevention of thrombosis, 9th ed: American College of Chest Physicians evidence-based clinical practice guidelines. Chest. 2012;141:e278S-e325S.

2. Eriksson BI, Borris LC, Friedman RJ, et al. Rivaroxaban versus enoxaparin for thromboprophylaxis after hip arthroplasty. NEngl J Med. 2008;358:2765-2775

3. Lassen MR, Ageno W, Borris LC, et al. Rivaroxaban versus enoxaparin for thromboprophylaxis after total knee arthroplasty. $N$ Engl J Med. 2008;358:2776-2786

4. Turpie AGG, Lassen MR, Davidson BL, et al. Rivaroxaban versus enoxaparin for thromboprophylaxis after total knee arthroplasty (RECORD4): a randomised trial. Lancet. 2009;373:1673-1680.

5. Turpie AGG, Lassen MR, Eriksson BI, et al. Rivaroxaban for the prevention of venous thromboembolism after hip or knee arthroplasty. Pooled analysis of four studies. Thromb Haemost. 2011;105:444-453.

6. Kakkar AK, Brenner B, Dahl OE, et al. Extended duration rivaroxaban versus short-term enoxaparin for the prevention of venous thromboembolism after total hip arthroplasty: a double-blind, randomised controlled trial. Lancet. 2008;372:31-39. 
7. Turpie AGG, Haas S, Kreutz R, et al. A non-interventional comparison of rivaroxaban with standard of care for thromboprophylaxis after major orthopaedic surgery in 17,701 patients with propensity score adjustment. Thromb Haemost. 2014;111:94-102.

8. Rosencher N, Bonnet MP, Sessler DI. Selected new antithrombotic agents and neuraxial anaesthesia for major orthopaedic surgery: management strategies. Anaesthesia. 2007;62:1154-1160.

9. Turpie AGG, Bauer KA, Eriksson BI, et al; Steering Committees of the Pentasaccharide Orthopedic Prophylaxis Studies. Fondaparinux vs enoxaparin for the prevention of venous thromboembolism in major orthopedic surgery: a meta-analysis of 4 randomized double-blind studies. Arch Intern Med. 2002;162:1833-1840.

10. Rodgers A, Walker N, Schug S, et al. Reduction of postoperative mortality and morbidity with epidural or spinal anaesthesia: results from overview of randomised trials. Br Med J. 2000;321:1493.

11. European Medicines Agency. Guideline on Clinical Investigation of Medicinal Products for Prevention of Venous Thromboembolism (VTE) in Patients Undergoing High VTE-Risk Surgery. 2012. Available from: http://www.ema.europa.eu/docs/en_GB/document_library/ Scientific_guideline/2012/05/WC500127902.pdf. Accessed January 20, 2016.

12. Raskob GE, Hirsh J. Controversies in timing of the first dose of anticoagulant prophylaxis against venous thromboembolism after major orthopedic surgery. Chest. 2003;124:379S-385S.

13. Bayer Pharma AG. Xarelto ${ }^{\circledR}$ (Rivaroxaban) Summary of Product Characteristics. 2015. Available from: http://www.ema.europa. eu/docs/en_GB/document_library/EPAR_-_Product_Information/ human/000944/WC500057108.pdf. Accessed January 20, 2016.
14. Gogarten W, Vandermeulen E, Van Aken H, et al; European Society of Anaesthesiology. Regional anaesthesia and antithrombotic agents: recommendations of the European Society of Anaesthesiology. Eur $J$ Anaesthesiol. 2010;27:999-1015.

15. Rosencher N, Llau JV, Mueck W, Loewe A, Berkowitz SD, Homering M. Incidence of neuraxial haematoma after total hip or knee surgery: RECORD programme (rivaroxaban vs. enoxaparin). Acta Anaesthesiol Scand. 2013;57:565-572.

16. Macfarlane AJ, Prasad GA, Chan VW, Brull R. Does regional anaesthesia improve outcome after total hip arthroplasty? A systematic review. Br J Anaesth. 2009;103:335-345.

17. Patel N, Khakha R, Gibbs J. Review article: anti-embolism stockings. J Orthop Surg (Hong Kong). 2013;21:361-364.

18. Ho KM, Tan JA. Stratified meta-analysis of intermittent pneumatic compression of the lower limbs to prevent venous thromboembolism in hospitalized patients. Circulation. 2013;128:1003-1020.

19. Kakkos SK, Warwick D, Nicolaides AN, Stansby GP, Tsolakis IA. Combined (mechanical and pharmacological) modalities for the prevention of venous thromboembolism in joint replacement surgery. $J$ Bone Joint Surg Br. 2012;94:729-734.

20. Weber JCP. Epidemiology of adverse reactions to nonsteroidal antiinflammatory drugs. In: Rainsford KD, Velo GP, editors. Advances in Inflammatory Research. New York: Raven Press; 1984:1-7.
Vascular Health and Risk Management

\section{Publish your work in this journal}

Vascular Health and Risk Management is an international, peerreviewed journal of therapeutics and risk management, focusing on concise rapid reporting of clinical studies on the processes involved in the maintenance of vascular health; the monitoring, prevention and treatment of vascular disease and its sequelae; and the involvement of

\section{Dovepress}

metabolic disorders, particularly diabetes. This journal is indexed on PubMed Central and MedLine. The manuscript management system is completely online and includes a very quick and fair peer-review system, which is all easy to use. Visit http://www.dovepress.com/ testimonials.php to read real quotes from published authors. 